\title{
Desenvolvimento de recursos didáticos para 0 ensino de Geociências para a Banca das Ciências e Experimentoteca da EACH/USP
}

\author{
Caroline Bertocco Garcia \\ Escola de Ciências, Artes e Humanidades - EACH-USP \\ Rosely Aparecida Liguori Imbernon \\ Escola de Artes, Ciências e Humanidades, EACH, \\ Univ. São Paulo USP, São Paulo / Programa de \\ Pós-graduação em Ensino e História das Ciências da \\ Terra, IG/Unicamp. \\ imbernon@usp.br \\ Ricardo Augusto V. Lacerda \\ Laboratório de Desenvolvimento de Recursos Didáti- \\ cos em Ciências da Natureza - LABDID-EACH-USP
}

\begin{abstract}
DEVELOPING TEACHING RESOURCES FOR GEOSCIENCES TEACHING FOR SCIENCE KIOSK AND EXPERIMENTS LIBRARY EACH/USP. This paper covers the importance of dissemination and popularization of science through the use of didactic models that express the contents of Geosciences. The dissemination and popularization of science has as a backdrop the Science Stand, a project of the Laboratory of Resource Development in Teaching Nature of Sciencem LABDID, in the School of Arts, Sciences and Humanities, EACH-USP. We used simple materials and activities presented in scientific publications in the field of education and Geosciences Geoideias to compose the collection of the Science Stand, as well as to expand the theme in the collection of the Geology in the Experimentoteca. The accessibility of materials for the construction of the models was seen as a means for the popularization of Geosciences, where choices of recycled and low cost remained dominant in the development and adaptation of models. Citation: Garcia C.B., Imbernon R.A.L., Lacerda R.A.V. 2014. Desenvolvimento de recursos didáticos para 0 ensino de Geociências para a Banca das Ciências e Experimentoteca da EACH/ USP. Terræ Didatica, 10(3):331-335. http://www.ige.unicamp.br/terraedidatica/.
\end{abstract}

KEYWORDS: Experimentation, Earth Learning Idea, Natural Sciences, Earth Sciences teaching.

RESUMO O presente trabalho abrange a importância da divulgação e popularização da Ciência com o uso de modelos didáticos que expressem os conteúdos de Geociências. A divulgação e popularização da Ciência tem como cenário a Banca da Ciência, um projeto ligado ao Laboratório de Desenvolvimento de Recursos Didáticos em Ciência da Natureza, LABDID da Escola de Artes, Ciências e Humanidades, EACH-USP. Foram utilizados materiais simples, como papel cartonado de caixa descartáveis, canudos para refrigerante, cola quente, copos de iogurte descartáveis etc. e atividades apresentadas em publicaçoes científicas da área de ensino de Geociências e do site Geoideias, para compor o acervo da Banca da Ciência, assim como para ampliar o tema Geologia no acervo da Experimentoteca do LABDID. A acessibilidade dos materiais para a construção dos modelos foi vista como um meio para a popularização das Geociências, na qual a escolha de materiais reciclados e de baixo custo permitisse a reprodução e adaptação dos modelos.

PALAVRAS-CHAVE: Experimentação, Geo-Ideias, Ciências Naturais, ensino de Ciências da Terra. 


\section{Introdução}

O objetivo do projeto Banca da Ciência é apresentar conceitos científicos de uma forma lúdica para o ensino formal e não formal. Para isso, o Laboratório de Desenvolvimento de Recursos Didáticos em Ciência da Natureza - LABDID da Escola de Artes, Ciências e Humanidades EACH-USP dispõe de um espaço de divulgação científica montado em uma estrutura de banca de jornal localizada dentro do campus da EACH-USP. Os grupos de estudantes e professores de Ciências agendam visitas monitoradas e nesse espaço aproveitam para discutir conceitos científicos e, principalmente, suas aplicações em nosso cotidiano. Além dos experimentos, a banca da Ciência tem em seu acervo publicações que vão desde livros e revistas científicas até obras de literatura infantil com aporte de conhecimentos científicos, principalmente conhecimentos que estão presentes no cotidiano do aluno.

A Experimentoteca, que até 2012 compunha o acervo da Estação Ciência da USP, passou a integrar o LABDID e tem como objetivo disponibilizar para professores do ensino básico kits didáticos da área de Ciências para utilização em sala de aula. Especificamente para o ensino de Geologia e Geociências, que compreendem conceitos teóricos e práticos abordados dentro do ensino formal de Geografia ou Ciências na educação básica, tanto a Banca quanto a Experimentoteca apresentam poucos temas. O conteúdo teórico proporciona sentindo e facilita o entendimento de um tema quando acompanhado de um modelo prático e interativo, de maneira que corresponda a um meio de troca de experiências entre aluno e professor e expresse o conteúdo teórico de forma acessível e simples.

A proposta é estimulada pela constatação de que os conteúdos disciplinares de Ciências da Terra apresentam-se fragmentados no ensino fundamental (Campos 1997). Carneiro et al. (2004) analisam a importância da contribuição da Geologia e da visão sistêmica para o ensino básico e assinalam que, de forma muito semelhante, de educação os conteúdos Geociências acham-se demasiadamente fragmentados no nível médio. De fato, ao nos remetermos aos Parâmetros Curriculares Nacionais (PCNs), identificamos conteúdos de Geociências, por exemplo, inseridos em disciplinas como Ciências da Natureza (ensino fundamental) e Geografia, Química (ensino médio). Compiani (2005) aponta que o currículo do ensino fundamental $\left(5^{\circ}\right.$ ao $9^{\circ}$ ano) tem ainda um currículo muito determinado e organizado em função das tradicionais disciplinas científicas, que se manteve mesmo após a implantação dos PCNs, promovendo o predomínio da "cultura disciplinar" que encontramos nas escolas em geral.

Os conteúdos relacionados à Geologia são tratados superficialmente em sala de aula, apenas vistos quando relacionados ao tema ar, água e solo. Questões evolutivas que envolvem os processos e a dinâmica terrestre são pouco aprofundadas pelos professores, que também não apresentam formação adequada para que sejam abordados de forma abrangente. Nesta perspectiva, Toledo (2005) discute a fragmentação da Geologia/Geociências em tópicos disseminados, muitas vezes, em diferentes disciplinas, que não contribui para a formação de uma noção do ciclo global da natureza (Carneiro et al. 2004).

Cabe ressaltar, também, as limitações dos livros didáticos, muitas vezes elaborados por docentes da área da Biologia e da Geografia, que não permitem a visão integrada das interações dos sistemas da terra (Barbosa 2003, Silva e Compiani 2002). Os fundamentos ambientais profundamente relacionados à física, seres vivos e as intenções entre os diferentes meios, envolvem problemáticas contemporâneas como a escassez de recursos minerais e o consumo de combustíveis fosseis (Campos, 1997).

A compreensão das mudanças que ocorrem no planeta, sejam pelos processos que envolvem a dinâmica terrestre, sejam pela ação antrópica interferindo nesses processos, influencia a maneira como vivemos; a formação de um cidadão crítico, que atua sobre o sistema Terra e compreende as consequências de sua atuação, que envolve o estimulo aos estudos em Ciências da Terra, e enfatiza a importância da Geologia/Geociências na formação da consciência ambiental para a preservação da natureza.

O desenvolvimento da autonomia do aluno permite que a mediação realizada pelo professor não se limite a transmitir informações, mas que ele/ ela apresente elementos de construção do conhecimento com a participação do aluno, passando da "informação" ao "conhecimento". A partir de suas (do aluno) escolhas e percepções do planeta, seus processos e dinâmica, o aluno, consequentemente, desenvolve um pensamento critico perante modelos sociais de uso e ocupação do planeta, que levaram aos grandes problemas ambientais que vivenciamos atualmente. Assim, buscamos 
desenvolver modelos didáticos que expressem os conteúdos de Geologia/Geociências, e apresentá-los em atividades de divulgação cientifica na Banca da Ciência, podendo ser utilizado como material complementar em sala de aula compondo a Experimentoteca disponível para professores do ensino público e privado.

\section{Metodologia}

Perante a necessidade de alternativas para a melhor abordar os conteúdos de Geologia/Geociências na escola, foi idealizado o projeto de elaboração de materiais para a divulgação cientifica por meio de modelos didáticos feitos com materiais acessíveis e de baixo custo que poderiam ser utilizados em oficinas infantis e em atividades desenvolvidas pelo professor em sala de aula.

O conceito de modelos didáticos para Geociências foi adaptado a partir dos modelos existentes no projeto Geo-Ideias desenvolvido pela iniciativa da equipe do Laboratório de Recursos Didáticos em Geociências (LRDG-DGAE), do IG-Unicamp, que se propôs a realizar a tradução/adaptação para o Português dos experimentos da série Earth Learning Idea.

A proposta de Geo-Ideias compartilha ideias inovadoras na criação de modelos de baixo custo que auxiliam professores com poucos recursos; os

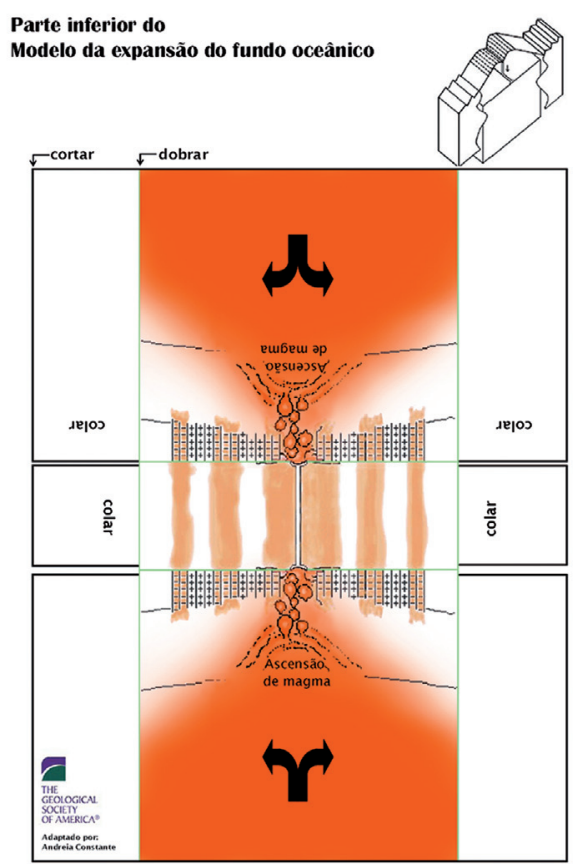

(1) autores abrem mão de direitos autorais para uso em laboratórios escolares e salas de aula (http://www. ige.unicamp.br/lrdg/geoideias.html).

Para a primeira etapa, que envolve temas para a Banca da Ciência e para a Experimentoteca, foram escolhidos alguns modelos para adaptação:

1) Modelos 3D da tectônica de placas (Constante e Vasconcelos, 2010): consiste em modelos de papel recortáveis em que o aluno cola e monta uma estrutura para a observação da interação das placas tectônicas, manuseando a representação da expansão do fundo oceânico e subdução (Fig. 1).

A adaptação realizada para a Banca da Ciência sugere um modelo principal de maior escala introduzindo um outro experimento complementar, com utilização de materiais diferentes. Nesse modelo de subdução foi introduzido o experimento do vulcão de sabão, inserido na área de vulcanismo gerado no processo de subdução (Figs. 2a e 2b).

A inserção desse segundo experimento no modelo, no qual o vulcão aparecia somente como ilustração, permite ao aluno interagir e perceber o vulcanismo como consequência da subdução .

O vulcão feito de garrafa pet inserido na estrutura representativa de subdução funciona a partir do sopro do aluno numa fina mangueira ligado a garrafa, fazendo com que o sabão misturado à água borbulhe simulando uma erupção. O modelo de

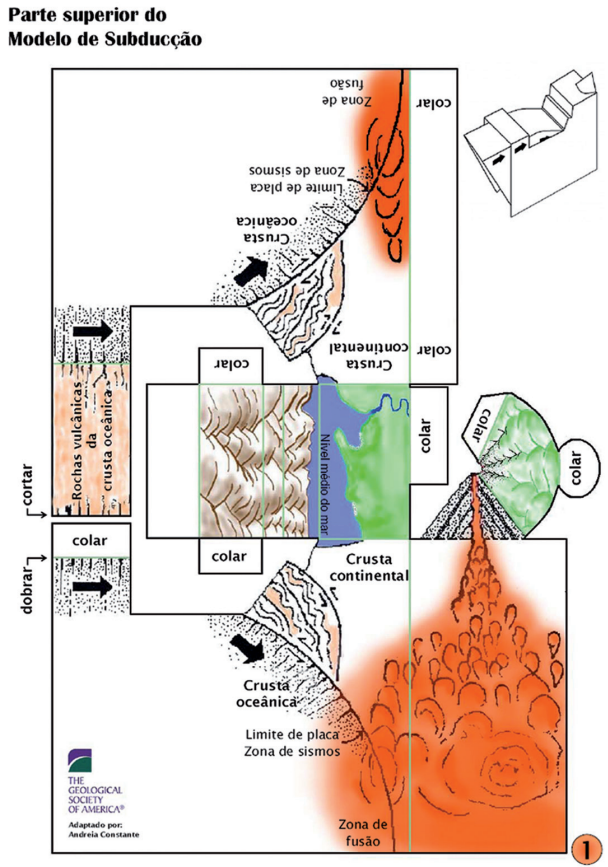

Figura 1. Modelo em papel para recortar e montar expansão oceânica em 3D (adaptado de Constante e Vasconcelos 2010) 

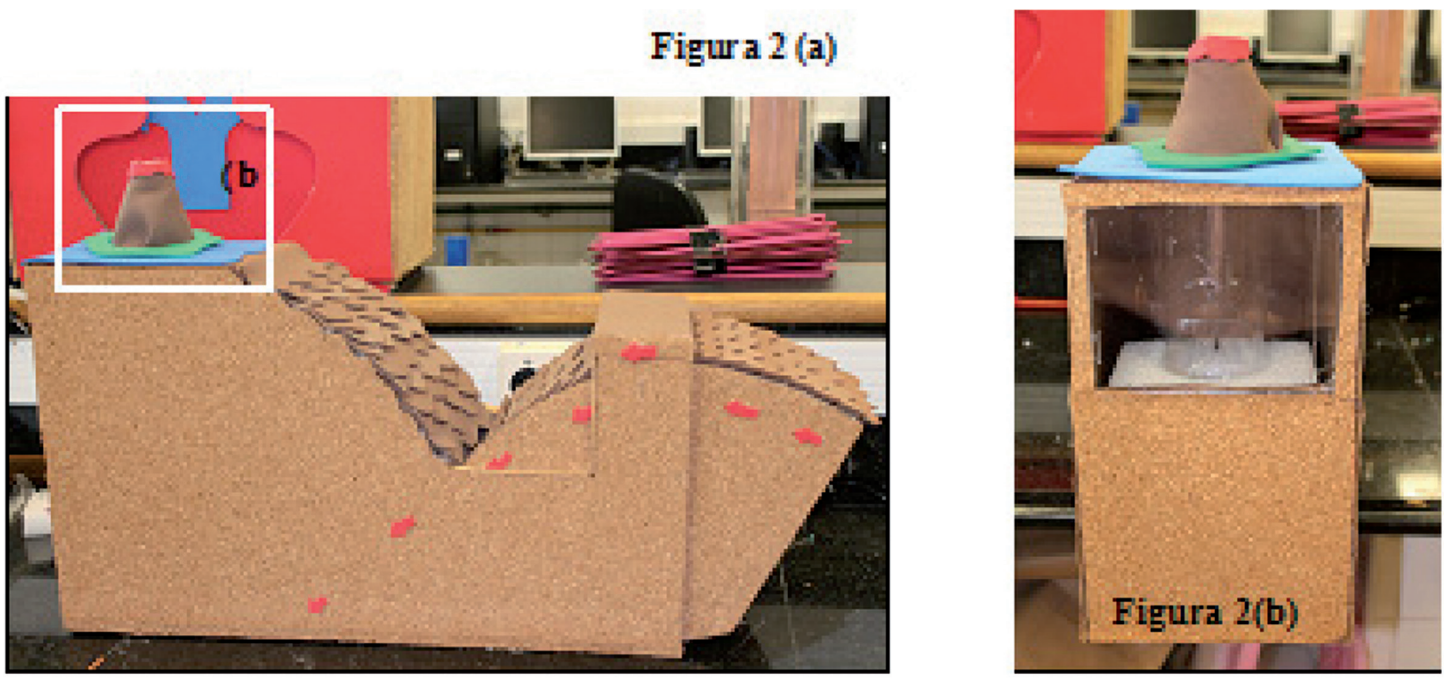

Figura 2. Modelo de subdução em tamanho macro (a) e detalhe (b) do vulcão gerado introduzido no modelo inicial

subdução foi adaptado com outros materiais mais consistentes como papelão e placas de EVA.

A atividade proposta é que, além do aluno montar o seu próprio modelo em papel, ele pode interagir com o modelo principal (em maior escala) exposto pelo professor, que explicará e solicitará ao aluno demonstrar com setas e placas o processo que ocorre em cada área do modelo.

2) A plasticidade da argila "splat" (Geoideias): diferente do modelo anterior, a "plasticidade da argila" não sofreu grandes modificações em sua adaptação do original. A proposta inicial era investigar a relação entre a plasticidade da argila e o teor adicionado de água durante o experimento, determinando essa relação com o diâmetro da mancha de barro "splat" causada pela queda da bola de argila de $50 \mathrm{~mm}$. A atividade permite introduzir conceitos sobre as propriedades da argila, mineralogia, além de permitir, também, a interdisciplinaridade com aplicação de conceitos da Física (elasticidade, plasticidade) e conteúdos de Matemática (cálculos de relação argila/água, construção de gráficos). $\mathrm{Na}$ adaptação foi elaborado um kit contendo argila seca, o suficiente para fazer uma bola de $50 \mathrm{~mm}$, fitas métricas de $2 \mathrm{~m}$, recipientes para água (com graduação), palitinhos para medição do diâmetro do splat, jornal, e papel milimetrado.

A dinâmica envolve que cada aluno receba uma porção de argila para fazer a bola de $50 \mathrm{~mm}$, em seguida os alunos adicionarão algumas gotas de água, que serão determinadas pelo professor em cada etapa da adição. Em cada etapa o aluno deverá soltar a bola de argila sobre o jornal e medir o diâmetro da mancha causada pela queda da bola úmida.

As medidas obtidas pelos alunos do diâmetro da mancha "splat", juntamente com as medidas adicionadas de água, servirão para a elaboração/ organização de uma tabela de forma que seja feito um gráfico para demonstrar os resultados e as observações dos alunos perante o experimento.

Os dois experimentos descritos foram destacados por terem sofrido maiores modificações perante o modelo original. Foram selecionados entre dez a quinze modelos que ainda estão em fase de adaptação e testes, e deverão, em função do tempo de execução, ser aplicados na Banca da Ciência ou incorporados ao acervo da Experimentoteca no tema Geologia.

\section{Conclusão}

A divulgação cientifica, como contribuição e proposta de ensino não formal, desempenha papel de extrema relevância durante o período de formação de cada individuo. É uma prática social que deve ser ampliada com auxílio de meios que permitam que os conteúdos sejam desenvolvidos com a participação em atividades que estimulem o pensamento crítico.

O desenvolvimento de práticas pedagógicas envolvendo a divulgação cientifica, que podem ser exploradas em espaços não formais de educação e em mídias, contribui para a popularização da Ciência.

O trabalho constitui proposta de divulgação e popularização das Geociências por meio de 
modelos didáticos adaptados, que podem ser utilizados em atividades que envolvam espaços formais de educação (em sala de aula), como material complementar para o professor (Experimentoteca), e também para o ensino não formal (Banca da Ciência) utilizados em oficinas promovidas em locais abertos onde o aprendizado é espontâneo.

\section{Referências bibliográficas}

Barbosa R. 2003. Projeto Geo-Escola: recursos computacionais de apoio a o ensino de geociências nos níveis fundamental e médio. Campinas: Inst. Geoc., Univ. Est. Campinas. 105p. (Dissert. Mestr.),

Campos D.A. 1997. O ensino das ciências da Terra. In: Simpósio a Importância da Ciência para o Desenvolvimento Nacional, 1, São Paulo. Do- cumentos... São Paulo: Acad. Bras. Ciências. p. 39-46.(1a. sessão, Educação).

Carneiro C.D.R., Toledo M.C.M.de; Almeida F.F.M. de. 2004. Dez motivos para a inclusão de temas de Geologia na Educação Básica. Rev. Bras. Geoc. 34(4):553-560. URL: sbgeo.org.br/pub_sbg/rbg/ vol34 down/3404/1439.PDF. Acesso 18.09.2014.

Compiani M. 2005. Geologia/Geociências no Ensino Fundamental e a Formação de Professores. São Paulo, Revista Geologia USP. Publ. Esp., 3:13-30.

Constante A., Vasconcelos C. 2010. Actividades lúdico-práticas no ensino da Geologia: complemento motivacional para a aprendizagem. Terre Didatica, 6(2):101-123.

Toledo M.C.M. 2005. Geociências no Ensino Médio Brasileiro. Análise dos Parâmetros Curriculares Nacionais. Revista Geologia USP. Publ. Esp., 3:33-34. 